\title{
Ortopedia prequirúrgica en el tratamiento de pacientes con fisura labio y paladar.
}

\section{Presurgical orthopedics in the treatment of patients with cleft lip and palate.}

\author{
Ignacio Domingo Agüero-Prado ${ }^{1 *}$, Matías Dallaserra ${ }^{2}$, Ignacio Araya ${ }^{2}$, Julio Villanueva ${ }^{2}$
}

\begin{abstract}
1. Facultad de Odontología, Universidad de Chile, Santiago, Chile.

2. Departamento de Cirugía y Traumatología Bucal y Maxilofacial, Facultad de Odontología, Universidad de Chile, Santiago, Chile.

* Correspondencia Autor: Ignacio Domingo Agüero Prado | Dirección: Facultad de Odontología Universidad de Chile. olivos 943, Independencia, Santiago, Chile | E-mail: ignacio@agüero.cl
\end{abstract}

\section{ORCID}

Ignacio Agüero-Prado: ORCID: 0000-0003-3378-8140 Matías Dallaserra: ORCID: 0000-0001-8654-1817 Ignacio Araya: ORCID: 0000-0003-2333-5033

Julio Villanueva: ORCID: 0000-0002-5807-442X

\section{RESUMEN}

Introducción: Dentro del tratamiento estándar de los pacientes con fisura labiomáxilo-palatinas se encuentra la ortopedia prequirúrgica. Esta se realiza con el objetivo de modular la posición, alineación y estabilización de los segmentos maxilares previo a la primera cirugía. Pese a que es ampliamente utilizada, su efectividad es controversial. Métodos: Se realizó una búsqueda en Epistemonikos, la mayor base de datos de revisiones sistemáticas en salud, la cual es mantenida mediante el cribado de múltiples fuentes de información, incluyendo MEDLINE, EMBASE, Cochrane, entre otras. Se extrajeron los datos desde las revisiones identificadas, se analizaron los datos de los estudios primarios, posteriormente se realizó un metaanálisis y se preparó una tabla de resumen de los resultados utilizando el método GRADE. Resultados y Conclusiones: Se identificaron 14 revisiones sistemáticas que en conjunto incluyeron 57 estudios primarios, de los cuales, cinco corresponden a ensayos clínicos aleatorizados. Concluimos que no es posible establecer con claridad si la ortopedia prequirúrgica mejora o perjudica la morfología maxilar, debido a que la certeza de la evidencia ha sido evaluada como muy baja. Por su parte, la ortopedia prequirúrgica podría resultar en poca o nula diferencia en el crecimiento y desarrollo facial, las alteraciones oclusales, la satisfacción parental, la alimentación, el habla y la apariencia facial y nasolabial, pero la certeza de la evidencia es baja.

PALABRAS CLAVE

Fisura labio-máxilo-palatina; Ortopedia prequirúrgica; Epistemonikos; GRADE.

\begin{abstract}
Introduction: Within the standard treatment of patients with labio-maxillo-palatal cleft is presurgical orthopedics. This procedure seeks to modulate the position, alignment and stabilization of the maxillary segments prior to the first surgery. Although it is widely used, its effectiveness is currently controversial. Methods: We searched in Epistemonikos, the largest database of systematic reviews in health, which is maintained by screening multiple information sources, including MEDLINE, EMBASE, Cochrane, among others. We extracted data from the systematic reviews, reanalyzed data of primary studies, conducted a meta-analysis and generated a summary of findings table using the GRADE approach. Results and conclusions: We identified 14 systematic reviews that included 57 primary studies, five of which correspond to randomized trials. We conclude that it is uncertain whether presurgical orthopedics improves or deteriorates maxillary morphology, because the certainty of the evidence has been very low. On the other hand, presurgical orthopedics may make little or no difference to facial growth and development, occlusal alterations, parental satisfaction, feeding, speech, and facial and nasolabial appearance, but the certainty of the evidence is low.

KEY WORDS
\end{abstract}

Labio-maxillo-palatal cleft; presurgical orthopedics; Epistemonikos; GRADE.

Int. J. Inter. Dent Vol. 14(2); 197-204, 2021. 


\section{PROBLEMA}

Las fisuras orofaciales se producen por una alteración en la fusión de los procesos embriológicos que eventualmente darán origen al labio, paladar o hueso alveolar durante el periodo de vida intrauterino. Se describen como de causa multifactorial ${ }^{(1,14)}$.

Las fisuras labio-máxilo-palatinas son las malformaciones congénitas más comunes que afectan al complejo maxilofacial, llegando a ocupar incluso hasta el tercer puesto de todas las malformaciones a nivel mundial(2). Según la Organización Mundial de la Salud (OMS), uno de cada 600 recién nacidos presenta esta condición ${ }^{(3)}$.

Las funciones vitales que se ven más comprometidas son la respiración, la deglución y la succión, además de generar consecuencias en la audición, cognición, habla, estética y en el ámbito psicosocial ${ }^{(4,5,6)}$ Debido a esto, el tratamiento de estos pacientes se aborda a través de un equipo de salud especializado multidisciplinario ${ }^{(5,79)}$.

Dentro de la amplia gama de tratamientos a los cuales son sometidos estos pacientes, se encuentra la ortopedia prequirúrgica. Este procedimiento es indicado de manera previa a las cirugías primarias con el objetivo de modular la posición, alineación y estabilización de los segmentos maxilares ${ }^{(7)}$

Pese a que la ortopedia prequirúrgica es ampliamente realizada, su efectividad se encuentra actualmente en controversia debido a la escasa disponibilidad de evidencia científica respecto a su efectividad y falta de evaluaciones de costo-efectividad a nivel mundial(1,5)

\section{MÉTODOS}

Realizamos una búsqueda en Epistemonikos, la mayor base de datos de revisiones sistemáticas en salud, la cual es mantenida mediante búsquedas en múltiples fuentes de información, incluyendo MEDLINE, EMBASE, Cochrane, entre otras. Extrajimos los datos desde las revisiones identificadas y analizamos los datos de los estudios primarios. Con esta información, generamos un resumen estructurado denominado FRISBEE (Friendly Summaries of Body of Evidence using Epistemonikos), siguiendo un formato preestablecido, que incluye mensajes clave, un resumen del conjunto de evidencia (presentado como matriz de evidencia en Epistemonikos), metanálisis del total de los estudios cuando sea posible, una tabla de resumen de resultados con el método GRADE y una sección de otras consideraciones para la toma de decisión.

\section{Mensajes clave}

- No es posible establecer con claridad si la ortopedia prequirúrgica mejora o perjudica la morfología maxilar, debido a que la certeza de la evidencia ha sido evaluada como muy baja.

- La ortopedia prequirúrgica podría resultar en poca o nula diferencia en el crecimiento y desarrollo facial, las alteraciones oclusales, la satisfacción parental, la alimentación, el habla y la apariencia facial y nasolabial (certeza de la evidencia baja).

Acerca del conjunto de evidencia para esta pregunta

Encontramos 14 revisiones sistemáticas ${ }^{(8-21)}$ que incluyen 39 estudios primario ${ }^{(22-78)}$ en

57 referencias, de las cuales cinco corresponden a ensayos aleatorizados ${ }^{(31,42,49,57,74)}$, reportados

en 21 referencias $(26,27,29,31,38$ 40,42,45,48,49,54,57,71-78) (17 referencias

Cuál es la evidencia Véase matriz de evidencia en Epistemonikos más abajo.

pertenecen al hilo de publicación "DUTCHCLEFT")

Esta tabla y el resumen en general se basan en estos últimos, dado que los estudios observacionales no aumentaban la certeza de la evidencia existente, ni entregaban información adicional relevante.
Todos los ensayos incluyeron recién nacidos de término, portadores de fisura labio-máxilo-palatina unilateral, no asociada a síndromes ni otra malformación congénita, excepto sindactilia $a^{(31,42,49,57,74)}$

Tres ensayos evaluaron los efectos del uso de la ortopedia prequirúrgica a través de placas de $\operatorname{Hotz}^{(31,42,49)}$.

Qué tipo de intervenciones incluyeron los estudios*

Los otros dos ensayos evaluaron los efectos del uso de la ortopedia prequirúrgica a través de modeladores nasoalveolar(57,74).

Los ensayos reportaron múltiples desenlaces, se escogieron para el análisis aquellos desenlaces que actualmente son controversiales en cuanto a la efectividad de la ortopedia los cuales fueron agrupados por las revisiones sistemáticas de la siguiente manera:

- Morfología maxilar

Qué tipo de desenlaces

- Crecimiento y desarrollo facial

- Alteraciones oclusales

- Satisfacción parental

- Alimentación

- Habla

- Apariencia facial y nasolabial

El seguimiento promedio de los ensayos fue de tres años con un rango que fluctuó entre los tres meses y 12 años $(31,42,49,57,74)$.

\begin{abstract}
* La información sobre los estudios primarios es extraída desde las revisiones sistemáticas identificadas, no directamente desde los estudios, a menos que se especifique lo contrario.
\end{abstract}

\section{RESUMEN DE LOS RESULTADOS}

El efecto estimado de la ortopedia prequirúrgica en pacientes con fisura labio-máxilo-palatina se basa en la información obtenida de cinco ensayos aleatorizados en los cuales se incluyeron 360 pacientes.

Dos ensayos midieron morfología maxilar (174 pacientes) ${ }^{(31,57)}$, uno midió crecimiento y desarrollo facial (54 pacientes) ${ }^{(31)}$, otro ensayo midió alteraciones oclusales (54 pacientes) ${ }^{(31)}$, uno midió satisfacción parental (54 pacientes) ${ }^{(31)}$, dos ensayos midieron alimentación (104 pacientes) $(31,42)$, uno midió habla (54 pacientes) ${ }^{(31)}$ y uno midió apariencia facial y nasolabial (54 pacientes) $)^{(31)}$.

El resumen de los resultados es el siguiente:

Morfología maxilar:

- No es posible establecer con claridad si la ortopedia prequirúrgica mejora o perjudica el ancho intercanino, debido a que la certeza de la evidencia existente ha sido evaluada como muy baja.

- No es posible establecer con claridad si la ortopedia prequirúrgica mejora o perjudica el ancho intertuberosidad, debido a que la certeza de la evidencia existente ha sido evaluada como muy baja.

- La ortopedia prequirúrgica podría resultar en poca o nula diferencia en la profundidad anteroposterior (baja certeza de evidencia).

\section{Crecimiento y desarrollo facial:}

- La ortopedia prequirúrgica podría resultar en poca o nula diferencia en el ángulo SNA (baja certeza de evidencia)

- La ortopedia prequirúrgica podría resultar en poca o nula diferencia en el ángulo SNB (baja certeza de evidencia).

- La ortopedia prequirúrgica podría resultar en poca o nula diferencia en el ángulo ANB (baja certeza de evidencia).

Alteraciones oclusales:

- La ortopedia prequirúrgica podría resultar en poca o nula diferencia 
en parámetros sagitales, verticales y transversales a los cuatro a seis años (baja certeza de evidencia).

- La ortopedia prequirúrgica podría resultar en poca o nula diferencia en parámetros transversales a los 12 años (baja certeza de evidencia).

\section{Satisfacción parental:}

- La ortopedia prequirúrgica podría resultar en poca o nula diferencia en la satisfacción parental (baja certeza de evidencia).

\section{Alimentación:}

- La ortopedia prequirúrgica podría resultar en poca o nula diferencia en el índice peso-talla (baja certeza de evidencia).

\section{Habla:}

- La ortopedia prequirúrgica podría resultar en poca o nula diferencia en el habla (baja certeza de evidencia).

\section{Apariencia facial y nasolabial}

- La ortopedia prequirúrgica podría resultar en poca o nula diferencia en la apariencia facial y nasolabial (baja certeza de evidencia)

\begin{tabular}{|c|c|c|c|c|}
\hline Pacientes & Fisuras Labio-Máxilo-Palatinas & & & \\
\hline $\begin{array}{l}\text { Intervención } \\
\text { Comparación }\end{array}$ & $\begin{array}{l}\text { Ortopedia prequirúrgica } \\
\text { Sin ortopedia prequirúrgica }\end{array}$ & & & \\
\hline \multirow{3}{*}{ Desenlaces } & & & \multirow{3}{*}{$\begin{array}{l}\text { Efecto } \\
\text { relativo } \\
\text { (IC 95\%) }\end{array}$} & \multirow{3}{*}{$\begin{array}{l}\text { Certeza de la evidencia } \\
\text { (GRADE) }\end{array}$} \\
\hline & $\begin{array}{c}\text { SIN } \\
\text { Ortopedia prequirúrgica } \\
\end{array}$ & $\begin{array}{c}\text { CON } \\
\text { Ortopedia prequirúrgica } \\
\end{array}$ & & \\
\hline & \multicolumn{2}{|c|}{ Diferencia: pacientes por 1000} & & \\
\hline \multicolumn{5}{|l|}{ Morfologia maxilar** } \\
\hline \multirow{2}{*}{ Ancho intercanino } & $26,86 \mathrm{~mm}$ & $28,39 \mathrm{~mm}$ & \multirow[b]{2}{*}{-} & \multirow[b]{2}{*}{$\underset{\text { Muy Baja }}{\oplus O O O^{1,2}}$} \\
\hline & \multicolumn{2}{|c|}{ DM: 1,52 mm más (Margen de error: -2,10 a 5,15) } & & \\
\hline \multirow{2}{*}{ Ancho intertuberosidad } & $40,66 \mathrm{~mm}$ & $39,79 \mathrm{~mm}$ & \multirow{2}{*}{-} & \multirow{2}{*}{$\begin{array}{l}\oplus O O O^{1,2} \\
\text { Muy Baja }\end{array}$} \\
\hline & DM: $0,7 \mathrm{~mm} \mathrm{~m}$ & $-3,47$ a 2,07$)$ & & \\
\hline \multirow{2}{*}{ Longitud maxilar anteroposterior } & $33,36 \mathrm{~mm}$ & $33,47 \mathrm{~mm}$ & \multirow[b]{2}{*}{-- } & \multirow{2}{*}{$\underset{\text { Baja }}{\oplus \oplus \mathrm{O}^{1}}$} \\
\hline & DM: 0,11 mm & $-1,79$ a 2,01$)$ & & \\
\hline \multicolumn{5}{|l|}{ Crecimiento y desarrollo facial $* * *$} \\
\hline \multirow[b]{2}{*}{ Ángulo SNA } & 83,43 grados & 82,14 grados & \multirow[b]{2}{*}{-} & \multirow{2}{*}{$\underset{\text { Baja }}{\oplus \oplus \mathrm{O}^{1}}$} \\
\hline & & & & \\
\hline \multirow{2}{*}{ Ángulo SNB } & 74,43 grados & 75,22 grados & \multirow{2}{*}{-} & \multirow{2}{*}{$\underset{\text { Baja }}{\oplus \oplus \mathrm{O}^{1}}$} \\
\hline & & & & \\
\hline \multirow{2}{*}{ Állgulu aivo } & 8,65 grados & 7,65 grados & \multirow[b]{2}{*}{-} & \multirow{2}{*}{$\underset{\text { Baja }}{\oplus \oplus \mathrm{OO}^{1}}$} \\
\hline & & & & \\
\hline \multicolumn{5}{|l|}{ Alteraciones oclusales } \\
\hline \multirow{2}{*}{$\begin{array}{l}\text { Parámetros oclusales sagitales, verticales y } \\
\text { transversales de cuatro a seis años }\end{array}$} & 0,13 puntos & 0,22 puntos & \multirow{2}{*}{-} & \multirow{2}{*}{$\underset{\text { Baja }}{\oplus \oplus \mathrm{OO}^{1}}$} \\
\hline & & & & \\
\hline \multirow{2}{*}{ Parámetros oclusales transversales a los 12 años } & $-3,6$ puntos & $-4,11$ puntos & \multirow{2}{*}{ - } & \multirow{2}{*}{$\underset{\text { Baja }}{\oplus \mathrm{OO}^{1}}$} \\
\hline & & & & \\
\hline Satisfacción & & & & \\
\hline Satisfacción parental & 1,5 puntos & 1,6 puntos & & $\oplus \oplus \mathrm{OO}^{1}$ \\
\hline & & & & \\
\hline Alimentación & & & & \\
\hline Pore Tall & $-0,36$ puntos & $-0,35$ puntos & & $\oplus \oplus \bigcirc^{1}$ \\
\hline Peso-Talla & & & - & Baja \\
\hline
\end{tabular}




\begin{tabular}{|c|c|c|c|c|}
\hline Habla & & & & \\
\hline \multirow{2}{*}{ Impresión total del habla } & 2,18 & 3,52 & \multirow{2}{*}{-} & \multirow{2}{*}{$\underset{\text { Baja }}{\oplus \bigcirc^{1}}$} \\
\hline & \multicolumn{2}{|c|}{ DM: 1,34 puntos más (Margen de error: 0,19 a 2,49) } & & \\
\hline \multicolumn{5}{|l|}{ Apariencia facial y nasolabial } \\
\hline \multirow{2}{*}{$\begin{array}{l}\text { Puntuaciones } z \text { de referencia y de escala visual } \\
\text { análoga agrupadas para fotografias de rostro } \\
\text { completo }\end{array}$} & $-0,03$ & 0,1 & \multirow{2}{*}{-} & \multirow{2}{*}{$\underset{\text { Baja }}{\oplus \oplus \mathrm{O}^{1}}$} \\
\hline & & & & \\
\hline \multirow{2}{*}{$\begin{array}{l}\text { Puntuaciones } z \text { de referencia y de escala visual } \\
\text { análoga agrupadas para fotografías recortadas }\end{array}$} & $-0,06$ & 0,12 & \multirow{2}{*}{-} & \multirow{2}{*}{$\oplus \underset{\text { Baja }}{\oplus O^{1}}$} \\
\hline & & & & \\
\hline \multicolumn{5}{|c|}{ 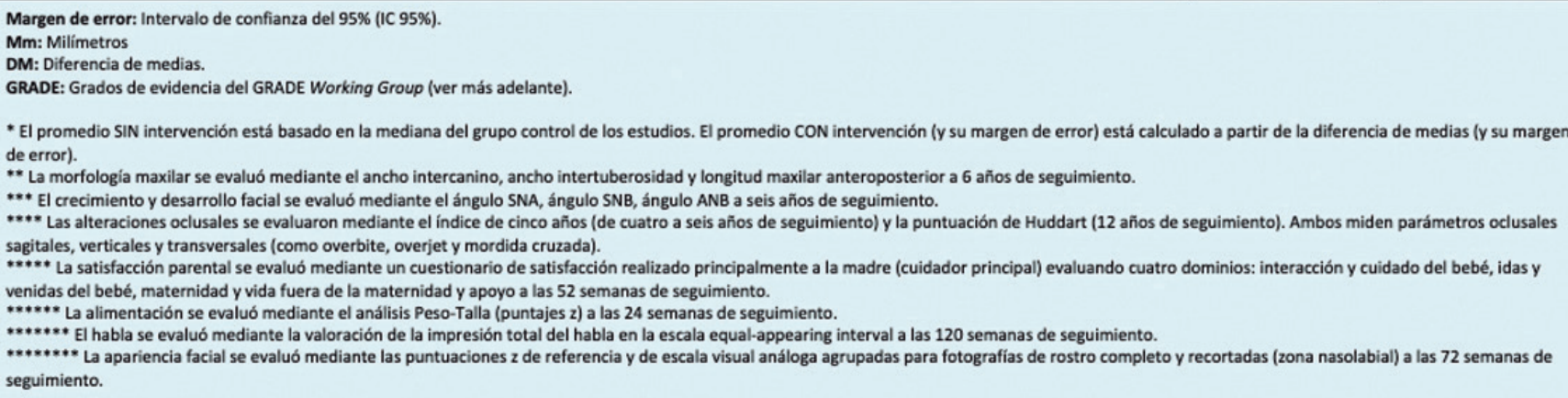 } \\
\hline \multicolumn{5}{|c|}{$\begin{array}{l}1 \text { Se disminuyeron dos niveles de certeza de evidencia por imprecisión, debido a que los intervalos de confianza fueron muy amplios y cada extremo del intervalo de confianza podría conllevar una decisión } \\
\text { diferente. Además, el tamaño muestral de los ensayos incluidos es muy pequeño. }\end{array}$} \\
\hline
\end{tabular}

Siga el enlace para acceder a la versión interactiva de esta tabla Interactive Summary of Findings - iSoF)

Acerca de la certeza de la evidencia (GRADE)*
$\bigoplus \bigoplus \bigoplus \bigoplus$
Alta: La investigación entrega una muy buena indicación del efecto probable. La probabilidad de
que el efecto sea sustancialmente distinto† es baja.
$\bigoplus \bigoplus \bigoplus \bigcirc$
Moderada: La investigación entrega una buena indicación del efecto probable. La probabilidad de
que el efecto sea sustancialmente distinto† es moderada.
$\bigoplus \bigoplus \bigcirc \bigcirc$
Baja: La investigación entrega alguna indicación del efecto probable. Sin embargo, la probabilidad
de que el efecto sea sustancialmente distinto† es alta.
$\bigoplus \bigcirc \bigcirc \bigcirc$
Muy baja: La investigación no entrega una estimación confiable del efecto probable. La
probabilidad de que el efecto sea sustancialmente distinto† es muy alta.
*Esto es también denominado 'calidad de la evidencia' o 'confianza en los estimadores del efecto'.
tSustancialmente distinto = una diferencia suficientemente grande como para afectar la decisión




\section{OTRAS CONSIDERACIONES PARA LA TOMA DE DECISIÓN}

\section{A quién se aplica y a quién no se aplica esta evidencia}

- La evidencia presentada en este resumen aplica a pacientes recién nacidos portadores de fisuras labio-máxilo-palatinas unilaterales que serán sometidos posteriormente a cirugías primarias.

- No aplica para pacientes portadores de fisuras labio-máxilopalatinas sindrómicas o relacionadas a otras malformaciones congénitas ni bandas de tejidos blandos, excepto sindactilia.

\section{Sobre los desenlaces incluidos en este resumen}

- Se incluyeron los desenlaces reportados en las revisiones sistemáticas los cuales fueron priorizados dada su relevancia clínica para los pacientes y tratantes, de acuerdo a la opinión de los autores de este resumen.

- Por su parte, para los desenlaces morfología maxilar, crecimiento y desarrollo facial, y alteraciones oclusales se seleccionaron aquellos desenlaces sustitutos que aporten en mayor medida al análisis de estos desenlaces principales.

\section{Balance riesgo/beneficio y certeza de la evidencia}

- El conjunto de evidencia muestra que existe poca o nula diferencia en cuanto a los desenlaces priorizados con el uso de ortopedia prequirúrgica, pero la certeza de la evidencia es baja o muy baja.

- Considerando lo anterior, no se ha establecido un efecto beneficioso ni adverso de la ortopedia prequirúrgica por lo que no parece prudente incorporar esta intervención a los protocolos quirúrgicos como parte del tratamiento de los pacientes con fisura labio-máxilo-palatinas.

\section{Consideraciones de recursos}

- Dos revisiones incluidas en este resumen ${ }^{(49,75)}$ realizaron un análisis de costos asociados a dos intervenciones en particular ${ }^{(8,14)}$. Ambas sugieren que la rentabilidad del tratamiento con ortopedia prequirúrgica no es aceptable.

- Adicionalmente a que el costo fue estadísticamente significativo mayor para el grupo con ortopedia prequirúrgica, la duración de la cirugía de labios fue en parte similar a la del grupo control. Sin embargo, otras variables no fueron incluidas en su análisis, tales como la función o aspecto del orbicular de los labios y la facilidad de la cirugía.

- Por otro lado, la evaluación del costo desde la perspectiva del desarrollo del habla (impresión total de la calidad del habla a corto plazo) refirió una pequeña mejora, la cual fue significativa, a favor del tratamiento con ortopedia prequirúrgica, por lo que ambos sugirieron una rentabilidad aceptable.

- Por último, en esta síntesis de evidencia no se incluyeron estudios que midieran el costo efectividad de la intervención con QALY o DALY (calidad de vida). Estudios anteriores reportaron que la ortopedia prequirúrgica no es rentable en comparación al tratamiento quirúrgico sin esta.

- Por lo pronto, es incierto si se considera o no apropiado invertir recursos en el uso de ortopedia prequirúrgica, pero esta evidencia podría mantenerse o cambiar en vista a futuros estudios que analicen las relaciones costos/beneficios sobre las variables más relevantes en cuestión.

\section{Qué piensan los pacientes y sus tratantes}

- El impacto psicológico que provocan los pacientes con fisuras labiomáxilo-palatinas a sus padres se encuentra ampliamente documentado, sin embargo, el efecto psicológico que provoca a sus padres esta misma condición junto al tratamiento de ortopedia prequirúrgica no presenta análisis concluyentes.

- Una referencia(45) perteneciente al estudio DUTCHCLEFT(31) presenta evidencia en relación a factores psicosociales tempranos, donde se evaluó los efectos de la ortopedia prequirúrgica relacionada a la satisfacción parental. Dado que no es posible medir la calidad de vida del paciente a etapas tempranas de vida, es que se lleva a cabo esta medición a través de lo que piensan los padres, más específicamente en este estudio, la madre (cuidadora principal). De esta forma se midió la interacción y cuidado del bebé, idas y venidas del bebé, maternidad y vida fuera de la maternidad y por último, el apoyo, dando como resultado que la ortopedia prequirúrgica no influye en la satisfacción parental al año de vida.

- Por otro lado, poco o nada hay reportado acerca de lo que piensan los tratantes en relación al uso de la ortopedia prequirúrgica como tratamiento para pacientes con fisuras labio-máxilo-palatinas, pero si se sabe que es una práctica que hasta el día de hoy sigue siendo ampliamente utilizada pese a la escasa evidencia a favor que existe para esta intervención.

\section{Diferencias entre este resumen y otras fuentes}

- Las conclusiones obtenidas a partir de este resumen coinciden con las revisiones incluidas con respecto a que no se observa un efecto importante en la efectividad del uso de ortopedia prequirúrgica como tratamiento para pacientes con fisuras labio-máxilo-palatinas, en cuanto a las características de morfología maxilar, crecimiento y desarrollo facial, alteraciones oclusales, satisfacción parental, alimentación, habla y apariencia facial y nasolabial.

- En la guía de práctica clínica del Ministerio de Salud de Chile(79) se recomienda que el uso de la ortopedia prequirúrgica quede a criterio de la experiencia del cirujano, haciendo mención de que no existe evidencia a favor ni en contra del uso de esta misma.

- Situación similar se puede encontrar en la guía práctica "Early care services for babies born with cleft lip and/or palate" desarrollada por la European Cleft Organisation ${ }^{(80)}$, donde menciona explícitamente no entregar recomendaciones sobre el uso de la ortopedia prequirúrgica, quedando exclusivamente a criterio del tratante y los padres.

\section{¿Puede que cambie esta información en el futuro?}

- Debido al bajo y muy bajo nivel de certeza, es altamente probable que la evidencia pueda cambiar en el futuro.

- Se identificaron nueve revisiones sistemáticas ${ }^{(81-89)}$ en curso en la base de datos PROSPERO (International prospective register of systematic reviews) y cuatro ensayos clínicos ${ }^{(90-93)}$ en curso en la International Clinical Trials Registry Platform de la Organización Mundial de la Salud, los cuales podrían aportar información adicional relevante en relación a la efectividad del uso de la ortopedia prequirúrgica en pacientes con fisuras labio-máxilo-palatinas.

\section{CÓMO REALIZAMOS ESTE RESUMEN}

Mediante métodos automatizados y colaborativos recopilamos toda la evidencia relevante para la pregunta de interés y la presentamos en una matriz de evidencia.

\section{Siga el enlace para acceder a la versión interactiva: Ortopedia prequirúrgica en el tratamiento de pacientes con fisura labio y paladar}

\section{NOTAS}

Si con posterioridad a la publicación de este resumen se publican nuevas revisiones sistemáticas sobre este tema, en la parte superior de la matriz se mostrará un aviso de "nueva evidencia".

Luego de crear una cuenta en Epistemonikos, al guardar las matrices recibirá notificaciones automáticas cada vez que exista nueva evidencia que potencialmente responda a esta pregunta.

Este artículo es parte del proyecto síntesis de evidencia de Epistemonikos. Se elabora con una metodología preestablecida, siguiendo rigurosos estándares metodológicos y proceso de revisión por pares interno. Cada uno de estos artículos corresponde a un resumen, denominado FRISBEE (Friendly Summary of Body of Evidence using Epistemonikos), cuyo principal objetivo es sintetizar el conjunto de evidencia de una pregunta específica, en un formato amigable a los profesionales clínicos. Sus principales recursos se basan en la matriz de evidencia de Epistemonikos y análisis de resultados usando metodología GRADE. Mayores detalles de los métodos para elaborar este FRISBEE están descritos aquí (http://dx.doi.org/10.5867/ medwave.2014.06.5997)

La Fundación Epistemonikos es una organización que busca acercar la información a quienes toman decisiones en salud, mediante el uso de tecnologías. Su principal desarrollo es la base de datos Epistemonikos (www.epistemonikos.org).

\section{DECLARACIÓN DE CONFLICTOS DE INTERESES}

Los autores declaran no tener conflictos de intereses con la materia de este artículo.

\section{FUENTE DE FINANCIAMIENTO}

Fondo de Investigación, Facultad de Odontología, Universidad de Chile FIOUCH 19-04. 


\section{Bibliografía}

1. Martinez, GK. Perfil epidemiológico de los pacientes con fisuras labio-máxilo-palatina no sindrómicas atendidos en el Hospital San Borja Arriarán durante el período 2005-2015. Tesis Cirujano Dentista. Universidad de Chile. 2017. Disponible en: http://repositorio.uchile.cl/ handle/2250/146567

2. Bravo Rivera L, Muñoz Tobar D \& Torres Chianale F. Ortopedia prequirúrgica en niños fisurados: Reporte de caso clínico. Odontoestomatología. 2018,17(25), 53-9.

3. World Health Organization (WHO). Addressing the Global Challenges of Craniofacial Anomalies. Geneva, Switzerland: WHO; 2006.

4. Cortés J, Nino A, Sung H, \& Cortés P. Estrategia terapéutica en las fisuras labio-máxilo-palatinas: La aproximación funcional de Delaire. Rev Esp Cirug Oral y Maxilofac. 2002, 24,13-9.

5. Mossey PA, Little J, Munger RG, Dixon MJ \& Shaw WC. Cleft lip and palate. Lancet. 2009,374(9703),1773-85.

6. Márquez MFL. Aspectos psicológicos de pacientes que presentan hendidura labio palatina. Acta Odontol Venez. 2013,51(2). Disponible en: https://www.actaodontologica.com/ediciones/2013/2/art-16/

7. Latham R, Latham R. Orthopedics advancement of the cleft maxillary segment: A preliminary report. Cleft Palate J. 1980;17(3):227-33.

8. Papadopoulos MA, Koumpridou EN, Vakalis ML, Papageorgiou SN Effectiveness of pre-surgical infant orthopedic treatment for cleft lip and palate patients: a systematic review and meta-analysis. Orthod Craniofac Res. 2012;15(4):207-36.

9. Uzel A, Alparslan ZN. Long-term effects of presurgical infant orthopedics in patients with cleft lip and palate: a systematic review. Cleft Palate Craniofac J. 2011;48(5):587-95.

10. Niranjane PP, Kamble RH, Diagavane SP, Shrivastav SS, Batra P, Vasudevan SD, et al. Current status of presurgical infant orthopaedic treatment for cleft lip and palate patients: A critical review. Indian J Plast Surg. 2014;47(3):293-302.

11. de Ladeira PR, Alonso N. Protocols in cleft lip and palate treatment: systematic review. Plast Surg Int. 2012;2012:562892.

12. Murthy PS, Deshmukh S, Bhagyalakshmi A, Srilatha K. Pre surgical nasoalveolar molding: changing paradigms in early cleft lip and palate rehabilitation. J Int Oral Health. 2013;5(2):70-80.

13. Bessell A, Hooper L, Shaw WC, Reilly S, Reid J, Glenny AM. Feeding interventions for growth and development in infants with cleft lip, cleft palate or cleft lip and palate. Cochrane Database Syst Rev. 2011;(2):CD003315.

14. Hosseini HR, Kaklamanos EG, Athanasiou AE. Treatment outcomes of pre-surgical infant orthopedics in patients with non-syndromic cleft lip and/or palate: A systematic review and meta-analysis of randomized controlled trials. PLoS One. 2017;12(7):e0181768.

15. Maillard S, Retrouvey JM, Ahmed MK, Taub PJ. Correlation between Nasoalveolar molding and surgical, aesthetic, functional and socioeconomic outcomes following primary repair surgery: a systematic review. J Oral Maxillofac Res. 2017;8(3):e2

16. van der Heijden P, Dijkstra PU, Stellingsma C, van der Laan BF, Korsten-Meijer AG, Goorhuis-Brouwer SM. Limited evidence for the effect of presurgical nasoalveolar molding in unilateral cleft on nasal symmetry: a call for unified research. Plast Reconstr Surg. 2013;131(1):62e-71e.

17. Bittermann GK, de Ruiter AP, Janssen NG, Bittermann AJ, van der Molen AM, van Es RJ, et al. Management of the premaxilla in the treatment of bilateral cleft of lip and palate: what can the literature tell us? Clin Oral Investig. 2016 Mar;20(2):207-17.

18. Abbott MM, Meara JG. Nasoalveolar molding in cleft care: is it efficacious? Plast Reconstr Surg. 2012;130(3):659-66.

19. Namdar P, Lal Alizadeh F, Etezadi T, Sadri L, Shiva A. Effect of nasoalveolar molding on nasal symmetry in patients with cleft lip and palate: a systematic review. J Pediatr Rev. 2020;8(2):79-92. https://doi. org/10.32598/jpr.8.2.79

20. Thierens L, Brusselaers N, De Roo N, De Pauw G. Effects of labia adhesion on maxillary arch dimensions and nasolabial esthetics in cleft lip and palate: a systematic review. Oral Dis. 2017;23(7):889-96.

21. Wahaj A, Ahmed I, Erum G. Nasal symmetry achieved by nasoalveolar molding in cleft lip and palate patients- a literature review. J Pak Dent Assoc. 2014;23(1):05-10

22. Suzuki K, Yamazaki Y, Sezaki K, Nakakita N. The effect of preoperative use of an orthopedic plate on articulatory function in children with cleft lip and palate. Cleft Palate Craniofac J. 2006;43(4):406-14.

23. Lee CT, Garfinkle JS, Warren SM, Brecht LE, Cutting CB, Grayson $\mathrm{BH}$. Nasoalveolar molding improves appearance of children with bilateral cleft lip-cleft palate. Plast Reconstr Surg. 2008;122(4):1131-7. 24. Liou EJ, Subramanian M, Chen PK, Huang CS. The progressive changes of nasal symmetry and growth after nasoalveolar molding: a three-year follow-up study. Plast Reconstr Surg. 2004;114(4):858-64. 25. Chan KT, Hayes C, Shusterman S, Mulliken JB, Will LA. The effects of active infant orthopedics on occlusal relationships in unilateral complete cleft lip and palate. Cleft Palate Craniofac J. 2003;40(5):5117.

26. Bongaarts CA, van 't Hof MA, Prahl-Andersen B, Dirks IV, Kuijpers-Jagtman AM. Infant orthopedics has no effect on maxillary arch dimensions in the deciduous dentition of children with complete unilateral cleft lip and palate (Dutchcleft). Cleft Palate Craniofac J. 2006;43(6):665-72.

27. Prahl C, Kuijpers-Jagtman AM, Van 't Hof MA, Prahl-Andersen B. A randomized prospective clinical trial of the effect of infant orthopedics in unilateral cleft lip and palate: prevention of collapse of the alveolar segments (Dutchcleft). Cleft Palate Craniofac J. 2003;40(4):337-42.

28. Pai BC, Ko EW, Huang CS, Liou EJ. Symmetry of the nose after presurgical nasoalveolar molding in infants with unilateral cleft lip and palate: a preliminary study. Cleft Palate Craniofac J. 2005;42(6):65863.

29. Prahl C, Kuijpers-Jagtman AM, van't Hof MA, Prahl-Andersen B. A randomised prospective clinical trial into the effect of infant orthopaedics on maxillary arch dimensions in unilateral cleft lip and palate (Dutchcleft). Eur J Oral Sci. 2001;109(5):297-305.

30. Mishima K, Mori Y, Sugahara T, Sakuda M. Comparison between the palatal configurations in complete and incomplete unilateral cleft lip and palate infants under 18 months of age. Cleft Palate Craniofac $\mathrm{J}$. 2001;38(1):49-54

31. Konst EM, Weersinkbraks $\mathrm{H}$. Prelexical development of unilateral cleft lip and palate babies with reference to presurgical infant orthopaedics: a randomized prospective clinical trial. Clinical Linguistics \& Phonetics. 1999;13(5):395-407.

32. Barillas I, Dec W, Warren SM, Cutting CB, Grayson BH. Nasoalveolar molding improves long-term nasal symmetry in complete unilateral cleft lip-cleft palate patients. Plast Reconstr Surg. 2009;123(3):1002-6.

33. Mishima K, Mori Y, Sugahara T, Minami K, Sakuda M. Comparison between palatal configurations in UCLP infants with and without a Hotz plate until four years of age. Cleft Palate Craniofac J. 2000;37(2):18590.

34. Baek SH, Son WS. Difference in alveolar molding effect and growth in the cleft segments: 3-dimensional analysis of unilateral cleft lip and palate patients. Oral Surg Oral Med Oral Pathol Oral Radiol Endod. 2006;102(2):160-8.

35. Maull DJ, Grayson BH, Cutting CB, Brecht LL, Bookstein FL, Khorrambadi $D$, et al. Long-term effects of nasoalveolar molding on three-dimensional nasal shape in unilateral clefts. Cleft Palate Craniofac J. 1999;36(5):391-7.

36. Spengler AL, Chavarria C, Teichgraeber JF, Gateno J, Xia JJ. Presurgical nasoalveolar molding therapy for the treatment of bilateral cleft lip and palate: A preliminary study. Cleft Palate Craniofac J. 2006;43(3):321-8

37. Hak MS, Sasaguri M, Sulaiman FK, Hardono ET, Suzuki A, Nakamura S, et al. Longitudinal study of effect of Hotz's Plate and lip adhesion on maxillary growth in bilateral cleft lip and palate patients. Cleft Palate Craniofac J. 2012;49(2):230-6.

38. Konst EM, Rietveld T, Peters HF, Kuijpers-Jagtman AM. Language skills of young children with unilateral cleft lip and palate following infant orthopedics: a randomized clinical trial. Cleft Palate Craniofac J. 2003;40(4):356-62.

39. Bongaarts CA, Prahl-Andersen B, Bronkhorst EM, Spauwen $\mathrm{PH}$ Mulder JW, Vaandrager JM, et al. Effect of infant orthopedics on facial appearance of toddlers with complete unilateral cleft lip and palate (Dutchcleft). Cleft Palate Craniofac J. 2008;45(4):407-13.

40. Prahl C, Kuijpers-Jagtman AM, Van 't Hof MA, Prahl-Andersen B. Infant orthopedics in UCLP: effect on feeding, weight, and length: a randomized clinical trial (Dutchcleft). Cleft Palate Craniofac J. 2005;42(2):171-7.

41. Ross RB, MacNamera MC. Effect of presurgical infant orthopedics on facial esthetics in complete bilateral cleft lip and palate. Cleft Palate Craniofac J. 1994;31(1):68-73.

42. Masarei AG, Wade A, Mars M, Sommerlad BC, Sell D. A randomized control trial investigating the effect of presurgical orthopedics on feeding in infants with cleft lip and/or palate. Cleft Palate Craniofac J. 2007;44(2):182-93.

43. Karling J, Larson O, Leanderson R, Henningsson G. Speech in unilateral and bilateral cleft palate patients from Stockholm. Cleft Palate Craniofac J. 1993;30(1):73-7.

44. Peat JH. Effects of presurgical oral orthopedics on bilateral complete clefts of the lip and palate. Cleft Palate J. 1982;19(2):100-3. 
45. Prahl C, Prahl-Andersen B, Van't Hof MA, Kuijpers-Jagtman AM. Presurgical orthopedics and satisfaction in motherhood: a randomized clinical trial (Dutchcleft). Cleft Palate Craniofac J. 2008;45(3):284-8. 46. Singh GD, Levy-Bercowski D, Santiago PE. Three-dimensional nasal changes following nasoalveolar molding in patients with unilateral cleft lip and palate: geometric morphometrics. Cleft Palate Craniofac J. 2005;42(4):403-9.

47. Ezzat CF, Chavarria C, Teichgraeber JF, Chen JW, Stratmann RG, Gateno J, et al. Presurgical nasoalveolar molding therapy for the treatment of unilateral cleft lip and palate: a preliminary study. Cleft Palate Craniofac J. 2007;44(1):8-12.

48. Prahl C, Prahl-Andersen B, van 't Hof MA, Kuijpers-Jagtman AM. Infant orthopedics and facial appearance: a randomized clinical trial (Dutchcleft). Cleft Palate Craniofac J. 2006;43(6):659-64.

49. Severens JL, Prahl C, Kuijpers-Jagtman AM, Prahl-Andersen B. Short-term cost-effectiveness analysis of presurgical orthopedic treatment in children with complete unilateral cleft lip and palate. Cleft Palate Craniofac J. 1998;35(3):222-6.

50. Mishima K, Sugahara T, Mori Y, Sakuda M. Three-dimensional comparison between the palatal forms in infants with complete unilateral cleft lip, alveolus, and palate (UCLP) with and without Hotz's plate. Cleft Palate Craniofac J. 1996;33(3):245-51.

51. Lohmander A, Lillvik M, Friede H. The impact of early infant jaworthopaedics on early speech production in toddlers with unilateral cleft lip and palate. Clin Linguist Phon. 2004;18(4-5):259-84.

52. Rau A, Ritschl LM, Mücke T, Wolff KD, Loeffelbein DJ. Nasoalveolar molding in cleft care--experience in 40 patients from a single centre in Germany. PLoS One. 2015;10(3):e0118103.

53. Liou EJ, Chen PK, Huang CS, Chen YR. Orthopedic intrusion of premaxilla with distraction devices before alveolar bone grafting in patients with bilateral cleft lip and palate. Plast Reconstr Surg. 2004;113(3):818-26.

54. Konst EM. The effects of infant orthopaedics on speech and language development in children with unilateral cleft lip and palate. M. Sc. Thesis. The University of Nijmegen. 2002.

55. Clark SL, Teichgraeber JF, Fleshman RG, Shaw JD, Chavarria C $\mathrm{Kau} \mathrm{CH}$, et. al Long-term treatment outcome of presurgical nasoalveolar molding in patients with unilateral cleft lip and palate. J Craniofac Surg. 2011;22(1):333-6.

56. Levy-Bercowski D, Abreu A, DeLeon E, Looney S, Stockstill J, Weiler M, et al. Complications and solutions in presurgical nasoalveolar molding therapy. Cleft Palate Craniofac J. 2009;46(5):521-8.

57. Shetty V, Agrawal RK, Sailer HF. Long-term effect of presurgical nasoalveolar molding on growth of maxillary arch in unilateral cleft lip and palate: randomized controlled trial. Int J Oral Maxillofac Surg. 2017;46(8):977-87.

58. Rachwalski M, Pullan S, Parmar R, Shetty PN, Sharma P, \& Bonanthaya K. Nasal aesthetics in unilateral cleft lip and palate with and without presurgical nasoalveolar molding: a preliminary assessment. Int J Oral Maxillofac Surg. 2017;46(Suppl. 1):68.

59. Shen C, Yao CA, Magee W 3rd, Chai G, Zhang Y. Presurgical nasoalveolar molding for cleft lip and palate: the application of digitally designed molds. Plast Reconstr Surg. 2015;135(6):1007e-15e

60. López-Palacio AM, Cerón-Zapata AM, Gómez DF, Dávila-Calle AP, Ojalvo-Arias MA. Nasal changes with nasoalveolar molding in Colombian patients with unilateral cleft lip and palate. Pediatr Dent. 2012;34(3):239-44.

61. Yu Q, Gong X, Shen G. CAD presurgical nasoalveolar molding effects on the maxillary morphology in infants with UCLP. Oral Surg Oral Med Oral Pathol Oral Radiol. 2013;116(4):418-26.

62. Mishra B, Singh AK, Zaidi J, Singh GK, Agrawal R, Kumar V. Presurgical nasoalveolar molding for correction of cleft lip nasal deformity: experience from northern India. Eplasty. $2010 \mathrm{Jul}$ 23;10:e55. 63. Gomez DF, Donohue ST, Figueroa AA, Polley JW. Nasal changes after presurgical nasoalveolar molding (PNAM) in the unilateral cleft lip nose. Cleft Palate Craniofac J. 2012;49(6):689-700.

64. Grabowski R, Kopp H, Stahl F, Gundlach KK. Presurgical orthopaedic treatment of newborns with clefts--functional treatment with long-term effects. J Craniomaxillofac Surg. 2006;34(Suppl 2):34-44.

65. Van der Beek MC, Hoeksma JB, Prahl-Andersen B, Meijer R. Effects of lip adhesion and presurgical orthopedics on facial growth: an evaluation of four treatment protocols. J Biol Buccale. 1992;20(4):1916.

66. Keçik D, Enacar A. Effects of nasoalveolar molding therapy on nasal and alveolar morphology in unilateral cleft lip and palate. J Craniofac Surg. 2009;20(6):2075-80.

67. Nakamura N, Sasaguri M, Nozoe E, Nishihara K, Hasegawa H, Nakamura S. Postoperative nasal forms after presurgical nasoalveolar molding followed by medial-upward advancement of nasolabial components with vestibular expansion for children with unilateral complete cleft lip and palate. J Oral Maxillofac Surg. 2009;67(10):222231.

68. Deng XH, Zhai JY, Jiang J, Li F, Pei X, Wang HT. [A clinical study of presurgical nasoalveolar molding in infants with complete cleft lip and palate]. Zhonghua Kou Qiang Yi Xue Za Zhi. 2005;40(2):144-6. Chinese.

69. Kozelj V. Experience with presurgical nasal molding in infants with cleft lip and nose deformity. Plast Reconstr Surg. 2007;120(3):738-45. 70. Kim N-Y, Shin-Jae Lee S-J, Seung Hak Baek, SH. Effect of presurgical nasoalveolar molding (PNAM) appliance and cheiloplasty on alveolar molding of complete unilateral cleft lip and palate patients. Korean J Orthod. 2003;33:234-45. Korean.

71. Bongaarts CA, Kuijpers-Jagtman AM, van 't Hof MA, PrahlAndersen $B$. The effect of infant orthopedics on the occlusion of the deciduous dentition in children with complete unilateral cleft lip and palate (Dutchcleft). Cleft Palate Craniofac J. 2004;41(6):633-41.

72. Konst EM, Weersink-Braks $H$, Rietveld T, Peters $H$. An intelligibility assessment of toddlers with cleft lip and palate who received and did not receive presurgical infant orthopedic treatment. J Commun Disord. 2000;33(6):483-99; quiz 499-501.

73. Konst EM, Rietveld T, Peters HF, Prahl-Andersen B. Phonological development of toddlers with unilateral cleft lip and palate who were treated with and without infant orthopedics: a randomized clinical trial. Cleft Palate Craniofac J. 2003;40(1):32-9.

74. Liang Z, Yao J, Chen PKT, Zheng C, Yang J. Effect of presurgical nasoalveolar molding on nasal symmetry in unilateral complete cleft lip/palate patients after primary cheiloplasty without concomitant nasal cartilage dissection: early childhood evaluation. Cleft Palate Craniofac J. 2018;55(7):935-40.

75. Konst EM, Prahl C, Weersink-Braks H, De Boo T, Prahl-Andersen B, Kuijpers-Jagtman AM, et al. Cost-effectiveness of infant orthopedic treatment regarding speech in patients with complete unilateral cleft lip and palate: a randomized three-center trial in the Netherlands (Dutchcleft). Cleft Palate Craniofac J. 2004;41(1):71-7.

76. Bongaarts CA, Prahl-Andersen B, Bronkhorst EM, Prahl C, Ongkosuwito EM, Borstlap WA, et al. Infant orthopedics and facial growth in complete unilateral cleft lip and palate until six years of age (Dutchcleft). Cleft Palate Craniofac J. 2009;46(6):654-63.

77. Konst EM, Rietveld T, Peters HF, Weersink-Braks H. Use of a perceptual evaluation instrument to assess the effects of infant orthopedics on the speech of toddlers with cleft lip and palate. Cleft Palate Craniofac J. 2003 Nov;40(6):597-605.

78. Noverraz RL, Disse MA, Ongkosuwito EM, Kuijpers-Jagtman AM, Prahl C. Transverse dental arch relationship at 9 and 12 years in children with unilateral cleft lip and palate treated with infant orthopedics: a randomized clinical trial (DUTCHCLEFT). Clin Oral Investig. 2015;19(9):2255-65.

79. MINSAL. Guía Clínica AUGE: Fisura Labiopalatina. Ministerio de Salud Pública de Chile. 2015.

80. CEN \& ECO. Early care services for babies born with cleft lip and/ or palate. Cen. 2015. Consultado [01/10/2020] Disponible en: http:// europeancleft.org/wp-content/uploads/2016/02/CEN.pdf

81. Daratsianos N, Do-Quang C, Gölz L, \& Jäger A. Efficacy of early orofacial orthopaedics applied to patients with cleft lip and palate: a systematic review. PROSPERO;2013:CRD42013006510. Disponible en: https://www.crd.york.ac.uk/prospero/display_record. php? ID=CRD42013006510

82. Abd MA, Fayed M, El-Beialy A, Eid F, \& Elkordy S. Effect of alveolar and nasoalveolar molding on parents' satisfaction, nose esthetics and maxillary arch dimensions in infants with unilateral cleft lip and palate: a systematic review and meta-analysis. PROSPERO;2016:CRD42016043174. Disponible en: https://www.crd. york.ac.uk/prospero/display_record.php?ID=CRD42016043174

83. Agnihotry A, Innes N, \& Gill K. Naso-alveolar molding for individuals with cleft lip and cleft palate. PROSPERO;2016:CRD42016033286. Disponible en: https://www.crd.york.ac.uk/prospero/display_record. php?ID=CRD42016033286

84. Ceron AM, Tamayo C, \& Manrique RD. Comparison of threedimensional changes of nasal soft tissue and the maxillary arch in patients with bilateral cleft lip and palate treated with presurgical orthopedic therapy. Systematic review. PROSPERO;2017:CRD42017069500. Disponible en: https://www.crd.york.ac.uk/prospero/display_record. php?ID=CRD42017069500

85. Gontijo, S. Nasal tissues changes in individuals with unilateral cleft lip or unilateral cleft lip and palate treated with presurgical orthopedic therapy: a systematic review. PROSPERO;2018:CRD42018107909. 
Disponible en: https://www.crd.york.ac.uk/prospero/display_record. php? ID=CRD42018107909

86. Padovano, W, Skolnick, G, Snyder-Warwick A, Colditz G, \& Patel K. The effects of presurgical nasoalveolar molding (NAM) in children with orofacial clefts. PROSPERO;2019:CRD42019127097. Disponible en: https://www.crd.york.ac.uk/prospero/display_record. php?ID=CRD42019127097

87. Delgado, MP, Rossell P, Olivencia C, \& Ormeño, R. Presurgical NAM (nasoalveolar molding): an evaluation of a rational treatment and systematic review. Part I: bilateral cleft lip and palate. PROSPERO;2019:CRD42019134146. Disponible en: https://www.crd. york.ac.uk/prospero/display_record.php?ID=CRD42019134146

88. Chunrong L, Cao M, Jin Z, Wang X, \& Chen L. Long-term effects of presurgical nasoalveolar molding on unilateral complete cleft lip and palate: Systematic review and meta-analysis. PROSPERO;2020:CRD42020142026. Disponible en: https://www.crd. york.ac.uk/prospero/display_record.php?ID=CRD42020142026

89. Villanueva J, Araya I, Yanine N, Dallaserra, Matías Salazar J, Martinez $Y$, et al. Effectiveness of pre-surgical orthopedics in the treatment of patients with unilateral cleft lip and palate: systematic review and metanalysis. PROSPERO;2020:CRD42020147963.

Disponible en: https://www.crd.york.ac.uk/prospero/display_record. php?ID=CRD42020147963

90. Abd El-Ghafour M. Effect of novel (Modified Grayson) techniques for nasoalveolar molding and taping on parents' satisfaction and shortterm treatment outcomes in infants with unilateral complete cleft lip and palate: a randomized controlled trial. NCT0284519. 2016. Disponible en: https://clinicaltrials.gov/show/NCT02845193

91. Hegde S, \& Parveen S. 3-Dimensional (3D) assessment of effect of orthopedic treatment modalities in nonsyndromic unilateral cleft lip and palate (NSUCLP) patients. CTRI/2017/02/007964. 2017. Disponible en: http://www.ctri.nic.in/Clinicaltrials/pmaindet2.php?trialid=17214

92. Saad, M. Efficacy of nasoalveolar molding therapy for treatment of unilateral cleft lip and palate in Egyptian infants. NCT03029195. 2017. Disponible en: https://clinicaltrials.gov/show/NCT03029195

93. Menezes, L. Effects of maxillary protraction with facemask on class iii orthopedic treatment between patients with and without complete unilateral cleft lip and palate through tomographic comparison. 2020. RBR-4fvrqx. Disponible en: https://apps.who.int/trialsearch/Trial2. aspx?TriallD=RBR-4fvrqx 